\title{
Diskriminierungserfahrungen und Bedrohungswahrnehmungen von in Deutschland lebenden Juden
}

\author{
Heiko Beyer · Ulf Liebe
}

Online publiziert: 5. Mai 2020

(C) Der/die Autor(en) 2020

Zusammenfassung Basierend auf Daten des Jahres 2018 der Fundamental Rights Agency der Europäischen Union ermittelt der vorliegende Beitrag Ausmaß und Faktoren antisemitischer Vorurteilskriminalität in Deutschland. Zum einen werden die Erfahrungen von in Deutschland lebenden Jüdinnen und Juden mit persönlichen Belästigungen und Beleidigungen, Vandalismus und körperlicher Gewalt innerhalb eines Zeitraumes von fünf Jahren vor dem Erhebungszeitpunkt beleuchtet. Zum anderen beschäftigen wir uns mit der geäußerten Furcht, zukünftig Opfer antisemitischer Übergriffe zu werden. Erfahrungen mit Gewalt und Vandalismus berichten $7 \%$ der 1225 Befragten, und $44 \%$ wurden in den letzten fünf Jahren belästigt, weil sie jüdisch sind. Vor allem Personen, die aufgrund des Tragens von Symbolen als Juden erkennbar sind, waren betroffen und vermeiden gelegentlich oder öfter Plätze in der lokalen Umgebung, weil sie sich dort unsicher fühlen. Wenn die Befragten hingegen in einer mehrheitlich jüdischen Nachbarschaft lebten, sank die Wahrscheinlichkeit Opfer von Belästigungen und Gewalttaten zu werden. Belästigt und beleidigt wurden zudem besonders religiöse Menschen und Personen, die die Unterstützung von Israel als sehr wichtig für ihre jüdische Identität erachten. Diese Personen fühlen sich, ebenso wie jene, die dem Erinnern an den Holocaust eine hohe Bedeutung beimessen, zudem stärker bedroht - eine Bestätigung der Vermutung, dass sekundärer und israelbezogener Antisemitismus ein großes Bedrohungspotential in der aktuellen gesellschaftlichen Situation darstellen. Als Reaktion auf die empfundene Bedrohung verzichten die Befragten zwar laut der vorliegenden Befragung nicht auf das Tragen von jüdischen Symbolen, aber stärkere Bedrohungswahrnehmungen

\footnotetext{
H. Beyer $(\square)$

Heinrich-Heine-Universität Düsseldorf, Düsseldorf, Deutschland

E-Mail: heiko.beyer@uni-duesseldorf.de

U. Liebe

University of Warwick, Coventry, Großbritannien

E-Mail: Ulf.Liebe@warwick.ac.uk
} 
korrelieren mit dem Vermeiden von als gefährlich eingeschätzten Plätzen sowie von jüdischen Veranstaltungen.

Schlüsselwörter Antisemitismus · Antizionismus · Judenfeindschaft · Vorurteilskriminalität · Hassrede

\title{
Discrimination experiences and threat perception of Jews living in Germany
}

\begin{abstract}
Based on data of the year 2018 from the Fundamental Rights Agency of the European Union, this article identifies the extent and factors of antisemitic hate crime in Germany. On the one hand, we examine experiences of Jews living in Germany regarding harassment, insults, vandalism and physical violence within a period of five years prior to the survey. On the other hand, we deal with the expressed fear of becoming victims of antisemitic attacks in the future. Experiences of violence and vandalism are reported by $7 \%$ of the sample $(n=1,225)$, and $44 \%$ have been harassed in the last five years because they are Jewish. In particular, people who are recognizable as Jews because they wear respective symbols were affected and at least occasionally avoid places in the local area because they feel unsafe there. If respondents lived in a predominantly Jewish neighborhood, the probability of becoming victims of harassment and acts of violence decreased. Incidents of harassment and insults were also reported more often by religious individuals and those respondents who consider support for Israel to be very important for their Jewish identity. These people, as well as those who attach great importance to Holocaust remembrance, also feel more threatened - confirming the assumption that secondary and Israel-related antisemitism represents a major threat in the current social situation. In response to the perceived threat, the interviewees do not, according to the present survey, refrain from wearing Jewish symbols, but stronger perceptions of threat correlate with the avoidance of places considered dangerous as well as with not participating in Jewish events.
\end{abstract}

Keywords Antisemitism - Antizionism · Jew hatred · Hate Crimes · Hate Speech

\section{Motivation und Forschungsstand}

Während die Anschläge auf die Hallenser Synagoge am 09. Oktober 2019, die zwei Menschen das Leben kosteten, bei der breiten Öffentlichkeit vor allem erstauntes Entsetzen und überraschte Bestürzung auslösten, kritisierten Vertreter*innen der jüdischen Gemeinde die mangelnden Sicherheitsvorkehrungen, auch angesichts des Wissens um das Gefahrenpotential antisemitischer Straftäter gerade an einem jüdischen Feiertag wie Jom Kippur, als ,skandalös“ (so Josef Schuster, der Vorsitzende des Zentralrats der Juden in Deutschland, siehe Tagesschau 2019). Wenngleich es sich bei dem bewaffneten Angriff von Halle, der darauf abzielte, alle 51 zur Tatzeit in der Synagoge Anwesenden zu töten, um den brutalsten Angriff auf jüdisches Leben 
in Deutschland seit 1945 handelt, reiht er sich ein in eine Reihe von antisemitischen Übergriffen der jüngeren Geschichte.

So stellt der Bericht des Kantor Centers (Porat 2018) zur weltweiten Verbreitung von Antisemitismus fest, dass die Zahl der antisemitischen Straftaten im Vergleich zum Vorjahr 2018 insbesondere in westlichen Ländern deutlich gestiegen sei. Dies gelte auch für Deutschland, wo vor allem die Häufigkeit von Gewalttaten zuletzt stark zunahm: 62 entsprechende Fälle wurden hier 2018 registriert - im Vergleich zum Vorjahr ein Anstieg um 70\%. Im Zeitraum zwischen 2001 und 2018 schwankte die Gesamtzahl der zur Anzeige gebrachten antisemitischen Straftaten in Deutschland zwischen 1239 Fällen im Jahr 2011 und 1809 Fällen im Jahr 2006 (Bundesregierung 2017). Im Jahr 2018 erreichte der Wert mit 1799 registrierten Fällen nahezu den Höchstwert von 2006 (Bundesministerium des Innern 2019). Mit Abstand am häufigsten handelt es sich um Propagandadelikte. Friedhofschändungen machten in etwa zwischen 30 und 60 der Straftatenfälle zwischen 2001 und 2013 aus. Die Anzahl der in diesem Zeitraum durch antisemitische Gewalt verletzten Personen liegt ebenfalls in diesem Bereich. Der überwiegende Teil der Straftaten hat einen rechtsextremistischen Hintergrund. Lediglich im Jahr 2009 überschritt der Anteil nichtrechts motivierter Straftaten die Zehn-Prozent-Marke (Bundesregierung 2017).

Das wachsende Risiko für in Deutschland lebende Jüdinnen und Juden drückt sich bei den Betroffenen in Verunsicherung und verstärkten Bedrohungswahrnehmungen aus. In einer im Jahr 2012 durchgeführten Online-Befragung im Auftrag der Agentur der Europäischen Union für Grundrechte (Fundamental Rights Agency, FRA 2013) wurden 5847 Jüdinnen und Juden mit einem Mindestalter von 16 Jahren aus 8 EUMitgliedsstaaten zu ihren Erfahrungen mit antisemitischen Übergriffen im Alltag befragt. In Deutschland gaben $44 \%$ der befragten Jüdinnen und Juden an, dass Antisemitismus in Deutschland ein großes Problem darstellt und 32\% waren der Ansicht, dass der Antisemitismus in Deutschland stark zugenommen hat. $24 \%$ gaben an, dass sie in den vorangegangenen 12 Monaten Zeuge von Vorfällen gewesen sind, bei denen Juden Opfer verbaler Beleidigung oder Belästigung und/oder körperlicher Gewalt wurden. $25 \%$ der deutschen Befragten berichteten zudem, aufgrund der wahrgenommenen Bedrohung durch Antisemitismus bereits über Auswanderung nachgedacht zu haben.

Eine zweite Studie der Agentur der Europäischen Union für Grundrechte (FRA 2018) aus dem Jahr 2018 bei der via Online-Befragung insgesamt 16.395 jüdische Menschen aus 12 EU-Mitgliedsstaaten zum Antisemitismus befragt wurden, ermittelte für die deutsche Teilstichprobe bereits einen Anteil von 60\% derer, die eine starke Zunahme des Antisemitismus im Land sehen. Der Anteil von Befragten, die in den vorangegangenen 12 Monaten in Deutschland Zeuge von Vorfällen geworden sind, bei denen Juden verbal beleidigt, belästigt und/oder körperlich angegriffen wurden, ist auf $29 \%$ gestiegen. Nunmehr berichteten $44 \%$, dass sie deshalb über eine Auswanderung aus Deutschland nachgedacht hätten.

Diese Zahlen decken sich weitgehend mit einer im Jahr 2017 veröffentlichten (Zick et al. 2017) und 2016 durchgeführten Studie des Instituts für interdisziplinäre Konflikt- und Gewaltforschung (IKG), im Rahmen derer 533 in Deutschland wohnhafte Menschen jüdischen Glaubens, die zum Befragungszeitpunkt älter als 15 Jahre alt waren, per Online-Survey befragt wurden. $76 \%$ davon halten Antisemitismus für 
ein großes Problem in Deutschland, wobei 69\% aufgrund persönlicher Erfahrungen zu dieser Einschätzung gelangten. 39\% waren der Ansicht, dass in Deutschland der Antisemitismus in den vergangenen 5 Jahren stark zugenommen hat.

Die (potentiellen) Täter*innen wurden in allen drei genannten Studien von den Befragten hauptsächlich im rechtsextremen, islamistischen sowie linken Milieu ausgemacht. Zick et al. (2017, S. 21) weisen zudem darauf hin, dass allerdings allein aufgrund der erhobenen Daten keine Aussagen darüber möglich sind, auf welcher Basis die Befragten auf die ideologischen Motive der Täter geschlossen haben.

Neben den skizzierten Ergebnissen standardisierter Befragungen liegen auch Ergebnisse einer qualitativen Teilstudie vor (Zick et al. 2017). Hierzu wurden 31 narrativ-biografische Interviews mit jüdischen Personen durchgeführt, die wichtige zusätzliche Einblicke liefern: Zum einen meiden die meisten Interviewpartner*innen seit 2014 häufig das Tragen jüdischer Symbole. Diejenigen, die sich zu erkennen geben, berichten von ,häufigen bis sehr häufigen“ Erfahrungen mit Antisemitismus. Zum anderen werden zwar unterschiedliche Akteure als Tätergruppe ausgemacht, aber in allen Interviews wird die mögliche Gefahr eines wachsenden Antisemitismus durch Geflüchtete aus muslimischen Ländern thematisiert. Gleichzeitig verursachte diese Einschätzung bei den Befragten ambivalente Gefühle, weil sie auch Mitleid mit den Geflüchteten empfanden. In den zusätzlichen 13 Experteninterviews mit Vertreterinnen und Vertretern jüdischer Verbände und Organisationen wurde diesbezüglich betont, dass islamistischer Antisemitismus nur eine von mehreren vorherrschenden Formen des Antisemitismus in Deutschland darstelle und davor gewarnt, Muslime allein für den Anstieg des Antisemitismus verantwortlich zu machen.

$\mathrm{Zu}$ einem ähnlichen Ergebnis kommt die Untersuchung „Flucht und Antisemitismus" des Berliner Instituts für empirische Integrations- und Migrationsforschung aus dem Jahr 2016 (Arnold und König 2016). Zahlreiche interviewte Expertinnen und Experten aus jüdischen Organisationen äußerten hier die Besorgnis, Muslime könnten pauschal als antisemitisch verurteilt werden und verwiesen auf das rassistische Potenzial einer solchen Pauschalisierung. Gleichzeitig betonten mehrere Expertinnen und Experten aber auch, dass aus ihrer Sicht die Gefahr, die vom islamistischen Antisemitismus für Jüdinnen und Juden ausgeht, in Öffentlichkeit und Politik noch nicht ausreichend ernstgenommen wird.

Der vorliegende Beitrag ergänzt die bisher existierenden Studien durch eine multivariate Betrachtung der Faktoren antisemitischer Gewalt- und Diskriminierungserfahrungen auf der einen Seite und Bedrohungswahrnehmungen auf der anderen Seite. Hierzu werden die Rohdaten der Fundamental Rights Agency (FRA) aus dem Jahr 2018 tiefergehend ausgewertet und diskutiert. Unsere Studie ergänzt damit die im Bericht der FRA bereits veröffentlichten Ergebnisse (vgl. FRA 2018) und untersucht sowohl die wahrgenommenen Ursachen persönlich erfahrener antisemitischer Gewalt und Belästigungen als auch die Faktoren entsprechender Bedrohungswahrnehmungen von in Deutschland lebenden jüdischen Menschen. Zu diesem Zweck werden multiple logistische Regressionsmodelle geschätzt, deren Ergebnisse im empirischen Teil unseres Artikels vorgestellt und diskutiert werden. Bevor wir zur Auswertung der Daten kommen, präzisieren wir nun zunächst die von uns verwendeten Begriffe und theoretischen Konzepte und formulieren empirisch prüfbare Hypothesen. 


\section{Begriffe, Theorien und Hypothesen}

\subsection{Diskriminierung und Vorurteilskriminalität}

Der Begriff Diskriminierung ist im Alltagsgebrauch meist mit dem ungleichen Zugang zu Ressourcen (zumeist via Arbeitsmarkt oder Bildung) verknüpft. Wir verwenden den Begriff hier in einer weiter gefassten Bedeutung, die solche Formen der Diskriminierung einschließt, aber ein breiteres Spektrum von Phänomenen bezeichnet, die zwischen den Polen verbaler Beschimpfung und körperlicher Gewalt bis hin zu Mord und Genozid verortet werden können (vgl. Marger 2011, S. 58). Eng verknüpft damit sind die Begriffe des ,hate crime“ (im deutschen „Hasskriminalität“ bzw. „Vorteilskriminalität“) sowie des ,hate speech“ (im deutsche „Hassrede“), die jeweils Teilmengen von Diskriminierungsphänomenen bezeichnen. Bei ,hate crimes" handelt es sich um kriminelle Handlungen, die zumindest partiell durch die wahrgenommene Gruppenzugehörigkeit des Opfers motiviert sind. In der Regel beziehen sich die Begriffe des ,hate crime“ und des „hate speech“ auf Vorurteile hinsichtlich der ethnischen Zugehörigkeit, der Religion, der sexuellen Orientierung oder dem Geschlecht der Opfer (vgl. Gerstenfeld 2013, S. 11 f.).

Merkmale von Hass- bzw. Vorurteilskriminalität im Vergleich zu anderen Gewaltverbrechen sind vor allem, dass erstere auf die zugeschriebene Gruppenidentität der Opfer zielt und neben der direkten auch eine symbolische Wirkung auf die Gesamtheit der entsprechenden Gruppe ausübt, indem die Mitglieder der Gruppe durch die Unberechenbarkeit der Tat verängstigt und eingeschüchtert werden sollen. Aufgrund der symbolischen Wirkung der Tat ist diese meist von besonderer Brutalität geprägt und versucht, den größtmöglichen Schaden an Personen und deren Eigentum sowie Plätzen der Identifikation zu verursachen (vgl. Coester 2015, S. 338). Das Spektrum der Hasskriminalität erstreckt sich von Vandalismus bis Mord (Gerstenfeld 2017, S. 159).

Während Taten die als Hasskriminalität interpretiert werden können an sich strafbar sind, gilt dies für Hassrede nicht in gleichem Maße (vgl. Gerstenfeld 2017, S. 159). Insbesondere in Ländern, in denen der Rede- und Meinungsfreiheit traditionell eine große Bedeutung eingeräumt wird, ist Hassrede in der Regel kein Straftatbestand. In Deutschland existiert umgekehrt keine allgemeine Hasskriminalitätsgesetzgebung - Richter*innen können bei der Strafzumessung aber Motive der Tat in Betracht ziehen - hingegen jedoch mit dem $\$ 130$ StGB der Tatbestand der Volksverhetzung, der als Äußerungsdelikt im Sinne einer Hassrede-Legislation interpretiert werden kann. Mit $§ 130$ Absatz 3 ist auch konkret der Straftatbestand der Holocaustleugnung bzw. -relativierung gesetzlich geregelt und damit Delikte der gegen jüdische Personen gerichteten Hassrede direkt justiziabel.

\subsection{Antisemitismus}

Phänomene der Holocaustleugnung bzw. -relativierung gehören zu den häufigsten und öffentlich sichtbarsten Formen des Antisemitismus nach 1945. Von der Antisemitismusforschung wird diese Dimension mit dem Begriff des ,Sekundären Antisemitismus“ erfasst (vgl. Schönbach 1961; Adorno 1997a, 1997b, 1997c; Rensmann 
1998, 2004). Dem Konzept liegt die Annahme eines psychofunktionalen Mechanismus zugrunde, bei dem die mit der sozialen Kategorie „Juden“ verknüpften Schuldund Aggressionsgefühle auf die Mitglieder jener Gruppe übertragen werden, indem die Rollen von Opfer und Täter verkehrt werden, „die Deutschen“ somit als Opfer ,jüdischer Rache“ erscheinen. In Bezug auf das politische System und das kulturelle Klima impliziert dies die „Kommunikationslatenz“ offenkundig antisemitischer Inhalte (Bergmann und Erb 1986): „Klassische“ antisemitische Aussagen wurden nach 1945 zunehmend öffentlich sanktioniert, um die Integration der Bundesrepublik in die westliche Staatengemeinschaft nicht zu gefährden. Als unmittelbar ,,antisemitisch“ zu erkennende Semantiken wurden in den Privatraum verdrängt oder auf die Kommunikation in Konsensgruppen (z. B. rechtsradikale Vereinigungen) beschränkt. Im öffentlichen Raum lösten Formen der „Umwegkommunikation“ (Bergmann und Erb 1986) offen-judenfeindliche Positionen ab. Sekundär-antisemitischen Positionen wie ,Reparationsforderungen an Deutschland nützen oft gar nicht den Opfern, sondern einer Holocaust-Industrie von findigen Anwälten." wird in Meinungsumfragen dementsprechend weitaus häufiger zugestimmt - bei der Umfrage von Decker et al. (2018, S. 200) aus dem Jahr 2018 z. B. von 36\% der Befragten - als „klassischen“ antisemitischen Items wie „Auch heute noch ist der Einfluss der Juden zu groß“ oder „Juden arbeiten mehr als andere mit üblen Tricks“ (10,1\% bzw. 7,6\% in der Studie von Decker et al. 2018, S. 193 f.).

Eine der populärsten Varianten sekundär-antisemitischer Umwegkommunikation stellt ,,israelbezogener Antisemitismus“ dar (auch unter dem Begriff des ,,neuen Antisemitismus“ diskutiert, so v. a. in der internationalen Debatte [vgl. Rabinovici et al. 2019] oder als „Antizionismus“ bezeichnet, so v.a. im Zusammenhang mit linkem Antisemitismus [vgl. Haury 2002; Poliakov 1992; Herf 2006]). Dabei wird Israel häufig mit Nazi-Deutschland gleichgesetzt, das Existenzrecht Israels angezweifelt und die Gruppe der Juden sowohl mit der Gruppe der Israelis als auch der Politik des Staates Israel identifiziert. Israelbezogener Antisemitismus ist Gegenstand kontroverser Diskussionen, vor allem in Bezug auf die Unterscheidung zwischen Fällen ausgewogener Kritik an der israelischen Politik auf der einen Seite und antisemitischen Generalisierungen und Stereotypisierungen auf der anderen Seite. Sharansky (2004) hat deshalb mit dem ,3D-Test“ ein Instrument vorgeschlagen, das dabei helfen soll, Diskurse und Aussagen auf ihren antisemitischen Gehalt zu überprüfen. Immer dann, wenn „Dämonisierungen“, „doppelmoralische“ Bewertungen oder eine „Delegitimierung“ der Existenz Israels stattfindet, könne von antisemitischen Motiven ausgegangen werden.

\subsection{Hypothesen}

Auf Basis der kurz dargestellten Literatur lassen sich grundlegende Hypothesen zu den Faktoren der Diskriminierungserfahrung und Bedrohungswahrnehmung jüdischer Personen ableiten. Schematisch können dabei kontextuelle von personenbezogenen Faktoren unterschieden werden. Aus der kriminologischen Forschung zu Hate Crimes können neben allgemeinen räumlichen und sozio-demografische Risikomerkmalen wie Urbanitätsgrad des Wohnorts und Alter der betroffenen Personen (vgl. Van Kesteren 2016) spezifische Faktoren von Vorurteilskriminalität gewonnen 
werden, so vor allem die Salienz der Gruppenzugehörigkeit und die Teilnahme an gruppenbezogenen Praxen (vgl. Hardy und Chakraborti 2020). Vor dem Hintergrund der Erkenntnisse der theoretischen und empirischen Forschung zum aktuellen Antisemitismus ergeben sich zudem Vermutungen über potentielle Tätergruppen und Tatmotive. Wir werden im Folgenden zunächst die Hypothesen in Bezug auf die Diskriminierungserfahrungen und im Anschluss daran im Hinblick auf die Bedrohungswahrnehmungen diskutieren.

\subsubsection{Diskriminierungserfahrung}

Da es sich bei verbalen oder körperlichen antisemitischen Übergriffen um gruppenbezogene Taten handelt, ist die Gefahr insbesondere für solche Personen gegeben, deren Zugehörigkeit zur Gruppe der „Juden“ salient ist. Das gilt sowohl für jene Menschen, die äußerlich z. B. durch das Tragen einer Kippa oder eines Davidsterns der Gruppe zugeordnet werden können als auch für jene, die aufgrund ihrer Religiosität Handlungen ausführen (Bräuche und Rituale) oder in Gesprächen Ansichten kommunizieren, die die jüdische Herkunft anzeigen. Dies gilt in gewissem Maße auch für Personen, die sich zwar nicht als sehr religiös verstehen, sich aber dennoch als Juden identifizieren. Die folgenden ersten Hypothesen ergeben sich aus diesen Überlegungen:

H1: Wenn Personen Symbole tragen, die sie als Jüdin/Jude erkennbar machen, dann ist es wahrscheinlicher, dass sie berichten, Opfer einer antisemitischen Straftat gewesen zu sein.

H2: Je religiöser jüdische Personen sind, desto wahrscheinlicher ist es, dass sie berichten, Opfer einer antisemitischen Straftat gewesen zu sein.

H3: Je ausgeprägter die jüdische Identität einer Person ist, desto wahrscheinlicher ist es, dass sie berichtet, Opfer einer antisemitischen Straftat gewesen zu sein.

Antisemitische Übergriffe werden laut offiziellen Statistiken in Deutschland am häufigsten von rechtsextremen Tätern begangen (Bundesregierung 2017). Dementsprechend ist anzunehmen, dass andere Elemente des Syndroms ,gruppenbezogener Menschenfeindlichkeit“ (Heitmeyer 2002) hinzutreten und entsprechende Gruppenmerkmale die Gefahr erhöhen, Opfer von rechter Gewalt zu werden. Insbesondere fremdenfeindliche und rassistische Motive dürften hier eine Rolle spielen. So ist etwa denkbar, dass jüdische Personen, die zwar in Deutschland leben, aber nicht in Deutschland geboren wurden und z.B. anhand ihrer Sprache als Migranten zu erkennen sind, zum einen häufiger diskriminiert und zum anderen häufiger auf ihre Herkunft angesprochen werden. Letzteres würde wiederum dazu führen, dass öfter ihr Jüdischsein salient gemacht würde. Als Tendenz lässt sich daher vermuten:

H4: Wenn jüdische Personen nach Deutschland zugewandert sind, dann ist es wahrscheinlicher, dass sie berichten, Opfer einer antisemitischen Straftat gewesen zu sein. 
Aus den Studien zum Sekundären Antisemitismus lässt sich entnehmen, dass Jüdinnen und Juden in Deutschland heute insbesondere dann mit verbaler und körperlicher Aggression rechnen müssen, wenn sie an die Verbrechen des Nationalsozialismus erinnern (vgl. als Überblick Salzborn 2019). Dies gilt für alle Mitglieder der Gruppe, da die Fremdzuschreibung in der Regel unabhängig vom Selbstverständnis der als „Juden“ identifizierten Personen vorgenommen wird. Für jene, die das Erinnern an den Holocaust als besonders wichtig erachten, dürfte sich das Risiko aber noch zusätzlich erhöhen, Ziel von antisemitischen Attacken zu werden.

H5: Je wichtiger es jüdischen Personen ist, die Erinnerung an den Holocaust zu bewahren, desto wahrscheinlicher ist es, dass sie berichten, Opfer einer antisemitischen Straftat gewesen zu sein.

Die zweite wichtige, damit verknüpfte Erkenntnis der Antisemitismusforschung besteht darin, dass Angriffe auf Jüdinnen und Juden heute sehr häufig damit gerechtfertigt werden, dass die israelische Politik Widerstand heraufbeschwöre (vgl. Hirsh 2013). Dabei kommt es den Tätern nicht unbedingt darauf an, welche konkrete Position das Opfer zu Israel oder zur israelischen Politik vertritt. Es findet auch hier wie generell beim Sekundären und Modernen Antisemitismus eine Personifizierung und Pauschalisierung statt (vgl. als Überblick Beyer 2015). Für jüdische Personen, die die Existenz Israels als sehr wichtig erachten, kann das Risiko, angegriffen und beleidigt zu werden, demnach als erhöht gelten.

H6: Je wichtiger es jüdischen Personen ist, Israel zu unterstützen, desto wahrscheinlicher ist es, dass sie berichten, Opfer einer antisemitischen Straftat gewesen zu sein.

Neben diesen personenbezogenen Faktoren antisemitischer Angriffe dürften räumliche Kontexte eine bedeutende Rolle spielen. Zunächst gilt wie bei allen Kriminalitätsdelikten, dass die Vermeidung von potentiellen Risikosituationen tendenziell weniger häufig zu Viktimisierungserfahrungen führt. Das würde im vorliegenden Fall bedeuten:

H7: Wenn jüdische Personen lokale Plätze meiden, die ihnen als unsicher für Juden erscheinen, dann ist es weniger wahrscheinlich, dass sie berichten, Opfer einer antisemitischen Straftat gewesen zu sein.

Weitere Kontextfaktoren lassen sich aus den spezifischen Charakteristika der Vorurteilskriminalität ableiten. So dürfte das Risiko antisemitischer Übergriffe in Nachbarschaften, in denen Juden die Mehrheit der Anwohner*innen darstellen, weitaus geringer sein, als in Stadtteilen oder Regionen, in denen nur wenige andere jüdische Menschen leben - zum einen aufgrund häufigerer sozialer Kontakte, die Vorurteile reduzieren können (vgl. Pettigrew und Tropp 2011) und zum anderen, weil hier die aus dem gesamtgesellschaftlichen Minderheitenstatus resultierende Vulnerabilität durch eine gestärkte Innergruppensolidarität teilweise kompensiert werden kann. 
H8: Wenn jüdische Personen in einer mehrheitlich jüdischen Wohngegend leben, dann ist es weniger wahrscheinlich, dass sie berichten, Opfer einer antisemitischen Straftat gewesen zu sein.

Für Veranstaltungen oder Orte der Identifikation gilt dies vermutlich jedoch nicht in gleichem Maße, da diese eine starke symbolische Bedeutung besitzen. Die Taten der Vorurteilskriminalität zielen aber gerade auf die symbolische Wirkung bei den Betroffenen (vgl. Coester 2015). Aus diesem Grund ist anzunehmen, dass Personen, die solche Veranstaltungen oder Orte der Identifikation nur selten oder gar nicht besuchen, weniger häufig von Straftaten betroffen sind.

H9: Wenn jüdische Personen ,jüdische“ Veranstaltungen oder Orte meiden, dann ist es weniger wahrscheinlich, dass sie berichten, Opfer einer antisemitischen Straftat gewesen zu sein.

\subsubsection{Bedrohungswahrnehmung}

Ein Großteil der geschilderten Zusammenhänge lässt sich auf das Phänomen der Bedrohungswahrnehmung übertragen, selbst wenn sich das Ausmaß der tatsächlichen Kriminalität und der empfundenen Bedrohung mitunter deutlich unterscheiden. Teilweise berichten sogar jene Gruppen, die, statistisch betrachtet, relativ selten Opfer von Kriminalität sind (z.B. alte Menschen), stärkere Ängste (Ziegler und Mitchell 2003). Kontrolliert man jedoch für die entsprechenden sozio-demografischen Variablen besteht ein positiver Zusammenhang zwischen eigenen Erfahrungen und Kriminalitätsfurcht (vgl. Stafford und Galle 1984). Im Fall antisemitischer Straftaten dürfte angesichts der real existierenden Bedrohung und der einschüchternden Wirkung von Vorurteilskriminalität eine entsprechende Korrelation ohnehin gegeben sein:

H10: Wenn jüdische Personen berichten, in der jüngeren Vergangenheit Opfer einer antisemitischen Straftat gewesen zu sein, dann ist die wahrgenommene Bedrohung durch antisemitische Angriffe ausgeprägter.

Nehmen wir weiterhin an, dass die Vermutungen des letzten Abschnitts und die entsprechenden Hypothesen angesichts der unmittelbaren persönlichen Betroffenheit auch von Jüdinnen und Juden selbst angestellt werden. Dies würde bedeuten, dass Personen, die sich bedroht fühlen, unter Umständen zum einen gerade verhindern wollen, als Jude erkannt zu werden und zum anderen Plätze meiden, die ihnen gefährlich erscheinen. Ebenso wäre allerdings denkbar, dass Personen, die sich nicht einschüchtern lassen wollen und auf solche Maßnahmen bewusst verzichten, deshalb größere Angst haben, Opfer von Übergriffen zu werden. Da unsere Annahmen im vorliegenden Artikel nur mit Querschnittdaten überprüft werden können, lassen sich diese konkurrierenden Kausalzusammenhänge nicht testen. Wir orientieren uns aus diesem Grund an den entsprechenden Hypothesen zur Diskriminierungserfahrung, betonen aber, dass die Auswertung an diesem Punkt eher explorativen Charakter 
hat. Die anderen Hypothesen können ohne diese Einschränkung übertragen werden: Menschen, denen ihre religiöse oder jüdische Identität sehr wichtig ist und die die Bedeutung des Holocaust-Gedenkens sowie jene Israels für die jüdische Identität als sehr wichtig einschätzen, dürften eher für das Problem des Antisemitismus sensibilisiert sein und sich dementsprechend stärker bedroht fühlen. Letzteres gilt nur für solche Personen nicht, die der Bundesregierung zutrauen, Antisemitismus effektiv zu bekämpfen - das heißt folglich:

H11: Je stärker jüdische Personen den Eindruck haben, dass die Regierung zu wenig gegen Antisemitismus unternimmt, desto ausgeprägter ist die wahrgenommene Bedrohung durch antisemitische Angriffe.

Die anderen Hypothesen lassen sich im Anschluss an die obigen Überlegungen folgendermaßen formulieren:

H1': Wenn Personen Symbole tragen, die sie als Jüdin/Jude erkennbar machen, dann ist es wahrscheinlicher, dass sie Bedrohungsgefühle artikulieren.

H2': Je religiöser jüdische Personen sind, desto wahrscheinlicher ist es, dass sie Bedrohungsgefühle artikulieren.

H3': Je ausgeprägter die jüdische Identität einer Person ist, desto wahrscheinlicher ist es, dass sie Bedrohungsgefühle artikuliert.

H4': Wenn jüdische Personen nach Deutschland zugewandert sind, dann ist es wahrscheinlicher, dass sie Bedrohungsgefühle artikulieren.

H5': Je wichtiger es jüdischen Personen ist, die Erinnerung an den Holocaust zu bewahren, desto wahrscheinlicher ist es, dass sie Bedrohungsgefühle artikulieren.

H6': Je wichtiger es jüdischen Personen ist, Israel zu unterstützen, desto wahrscheinlicher ist es, dass sie Bedrohungsgefühle artikulieren.

H7': Wenn jüdische Personen lokale Plätze meiden, die ihnen als unsicher für Juden erscheinen, dann ist es weniger wahrscheinlich, dass sie Bedrohungsgefühle artikulieren.

H8': Wenn jüdische Personen in einer mehrheitlich jüdischen Wohngegend leben, dann ist es weniger wahrscheinlich, dass sie Bedrohungsgefühle artikulieren.

H9': Wenn jüdische Personen ,jüdische“ Veranstaltungen oder Orte meiden, dann ist es weniger wahrscheinlich, dass sie Bedrohungsgefühle artikulieren. 


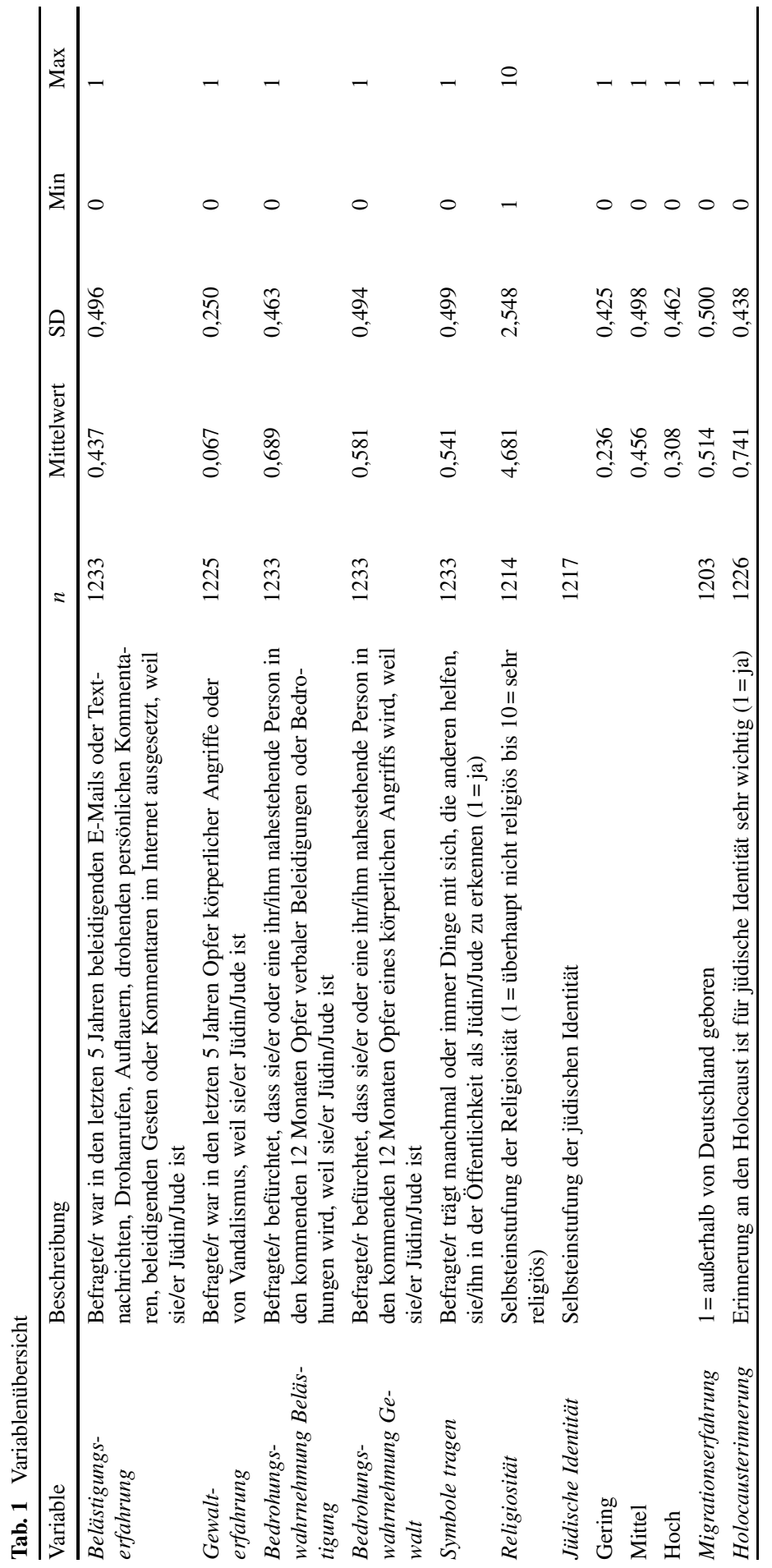




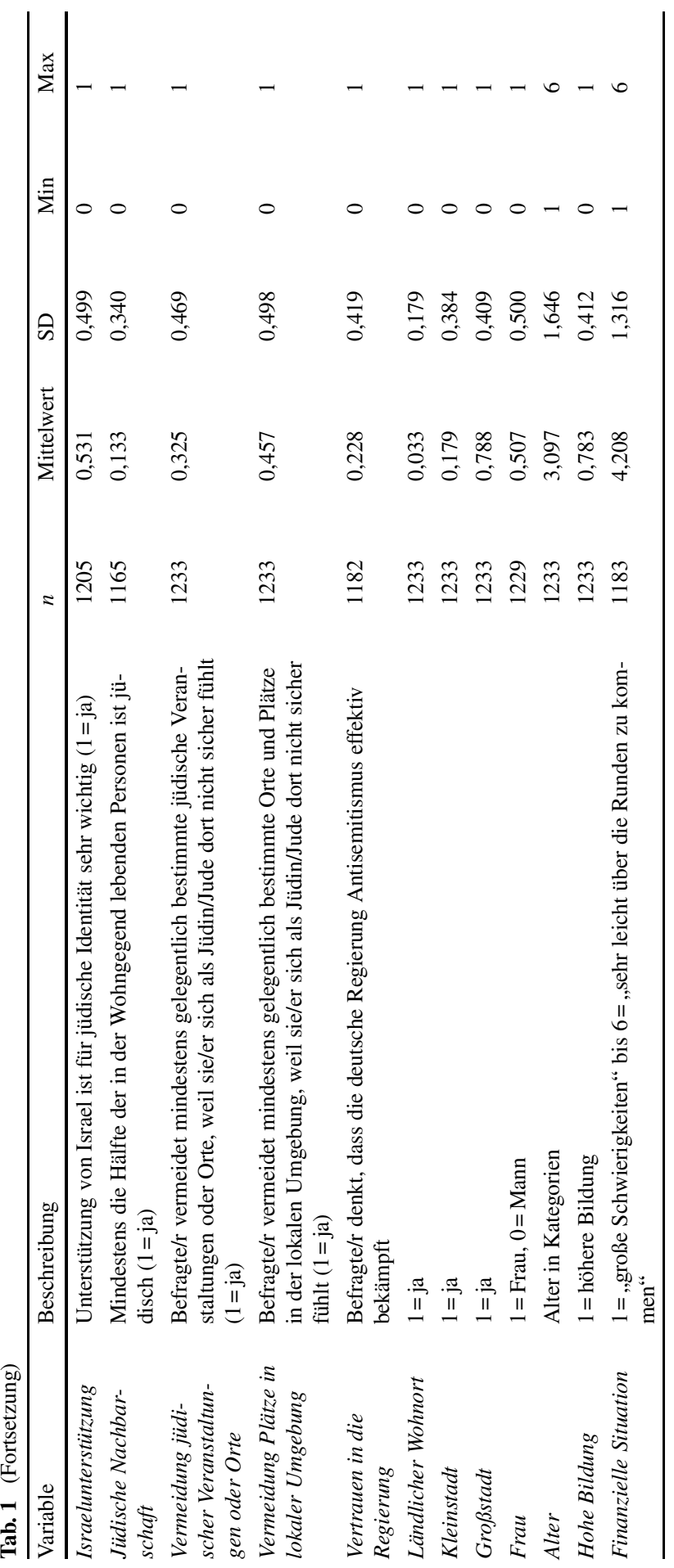




\section{Datensatz und Variablen}

Die Daten für die vorliegende Studie wurden im Mai und Juni 2018 durch die Agentur der Europäischen Union für Grundrechte (FRA) im Rahmen einer OnlineBefragung von in Deutschland lebenden Jüdinnen und Juden erhoben. Diese Datenerhebung war Teil einer, bereits zu Beginn dieses Aufsatzes erwähnten, größeren Studie zu Erfahrungen und Wahrnehmungen der jüdischen Bevölkerung in Bezug auf Hasskriminalität, Diskriminierung und Antisemitismus in 12 europäischen Ländern. In Deutschland haben 1233 Personen an der Befragung teilgenommen, wobei es sich um eine selektive und damit keine repräsentative Stichprobe handelt. Die FRA hatte Organisationen und Gruppen der Jüdischen Gemeinde gebeten, Einladungen zur Umfrage an ihre Mitglieder zu senden. Neben diesem Erstkontakt erfolgten noch zwei Erinnerungsemails. Zudem wurde auch mit Postern in lokalen jüdischen Zentren und jüdischen Medien auf die Umfrage aufmerksam gemacht. Somit haben die Befragten letztlich auf eigene Initiative hin an der Befragung (im Unterschied zu einer Zufallsstichprobe auf Basis von Mitgliederlisten etc.), die auf Deutsch oder Russisch beantwortet werden konnte, teilgenommen. Im Abgleich mit offiziellen statistischen Daten zur jüdischen Bevölkerung in Deutschland kommt die FRA zum Schluss, dass das Befragten-Sample der Studie die jüdische Bevölkerung in Deutschland moderat abbildet (siehe FRA 2019). Es liegen demnach Verzerrungen in der Stichprobe vor, die sich auf die Merkmale Gender (weniger Frauen in der Stichprobe), Alter (mehr ältere Personen), Bildung (überproportional höher Gebildete) und Wohnort (überproportional Berlin) beziehen. Dies sollte bei den deskriptiven Befunden berücksichtigt werden. In den multiplen Regressionsanalysen kontrollieren wir diese Merkmale.

Tabelle 1 gibt einen Überblick über die abhängigen und unabhängigen Variablen. Es zeigt sich, dass $44 \%$ der Befragten angegeben haben, in den vergangenen 5 Jahren mindestens einmal Hasskriminalität durch E-Mails, persönliche Kommentare, etc. erlebt zu haben; $7 \%$ berichten von Erfahrungen mit körperlichen Angriffen oder Vandalismus am eigenen Haus, Auto oder anderen Besitz (die beiden Deliktgruppen Körperverletzung und Sachbeschädigung haben wir aufgrund der niedrigen Fallzahlen für die späteren Analysen kombiniert; von Körperverletzungen berichten ca. 5\% und von Vandalismus 3\% der Befragten, wobei Mehrfachnennungen möglich waren). Bei diesen Angaben bleibt zu berücksichtigen, dass 5 Jahre ein langer Zeitraum sind, sodass Erinnerungsfehler und damit verbundene Verzerrungen in den

Tab. 2 Tätergruppen

\begin{tabular}{lll}
\hline & $\begin{array}{l}\text { Belästigung } \\
(n=647)\end{array}$ & Körperliche Gewalt $(n=63)$ \\
\hline Rechtsradikaler Hintergrund & 19,78 & 17,46 \\
Linksradikaler Hintergrund & 15,92 & 12,70 \\
Islamistischer Hintergrund & 41,42 & 60,32 \\
Bekannter oder Freund & 18,55 & 4,76 \\
Kollege oder Mitschüler/Kommilitone & 20,40 & 11,11 \\
Teenager & 18,55 & 20,63 \\
\hline
\end{tabular}

Anmerkungen: Angaben in Prozent; Mehrfachnennungen möglich 
Tab. 3 Multiple Logistische Regressionen zur Diskriminierungserfahrung und Bedrohungswahrnehmung

\begin{tabular}{|c|c|c|c|c|}
\hline & \multicolumn{2}{|c|}{ Diskriminierungserfahrung } & \multicolumn{2}{|c|}{ Bedrohungswahrnehmung } \\
\hline & Belästigung & $\begin{array}{l}\text { Gewalt/ } \\
\text { Vandalismus }\end{array}$ & Belästigung & $\begin{array}{l}\text { Gewalt/ } \\
\text { Vandalismus }\end{array}$ \\
\hline Symbole tragen & $\begin{array}{l}0,210 * * * \\
(7,16)\end{array}$ & $\begin{array}{l}0,049 * * \\
(3,23)\end{array}$ & $\begin{array}{l}0,035 \\
(1,37)\end{array}$ & $\begin{array}{l}0,014 \\
(0,50)\end{array}$ \\
\hline Religiosität & $\begin{array}{l}0,023 * * * \\
(3,93)\end{array}$ & $\begin{array}{l}0,001 \\
(0,29)\end{array}$ & $\begin{array}{l}0,012^{*} \\
(2,29)\end{array}$ & $\begin{array}{l}0,013 * \\
(2,21)\end{array}$ \\
\hline Jüd. Identität mittel & $\begin{array}{l}0,006 \\
(0,15)\end{array}$ & $\begin{array}{l}-0,033+ \\
(-1,70)\end{array}$ & $\begin{array}{l}0,028 \\
(0,93)\end{array}$ & $\begin{array}{l}0,035 \\
(0,99)\end{array}$ \\
\hline Jüd. Identität hoch & $\begin{array}{l}0,018 \\
(0,43)\end{array}$ & $\begin{array}{l}-0,027 \\
(-1,30)\end{array}$ & $\begin{array}{l}0,054 \\
(1,53)\end{array}$ & $\begin{array}{l}0,071+ \\
(1,79)\end{array}$ \\
\hline Migrationserfahrung & $\begin{array}{l}-0,085 * * \\
(-3,03)\end{array}$ & $\begin{array}{l}-0,012 \\
(-0,81)\end{array}$ & $\begin{array}{l}0,009 \\
(0,38)\end{array}$ & $\begin{array}{l}0,052+ \\
(1,93)\end{array}$ \\
\hline $\begin{array}{l}\text { Wichtigkeit Holocausterinne- } \\
\text { rung }\end{array}$ & $\begin{array}{l}-0,004 \\
(-0,11)\end{array}$ & $\begin{array}{l}0,014 \\
(0,81)\end{array}$ & $\begin{array}{l}0,101 * * * \\
(3,39)\end{array}$ & $\begin{array}{l}0,104 * * \\
(3,20)\end{array}$ \\
\hline Israelunterstützung & $\begin{array}{l}0,112 * * * \\
(3,67)\end{array}$ & $\begin{array}{l}-0,006 \\
(-0,36)\end{array}$ & $\begin{array}{l}0,071 * * \\
(2,64)\end{array}$ & $\begin{array}{l}0,100 * * * \\
(3,39)\end{array}$ \\
\hline $\begin{array}{l}\text { Jüd. Personen im Wohnum- } \\
\text { feld }\end{array}$ & $\begin{array}{l}-0,088^{*} \\
(-2,19)\end{array}$ & $\begin{array}{l}-0,048 * * \\
(-3,10)\end{array}$ & $\begin{array}{l}-0,035 \\
(-0,92)\end{array}$ & $\begin{array}{l}-0,017 \\
(-0,43)\end{array}$ \\
\hline $\begin{array}{l}\text { Vermeidung jüd. Veranstal- } \\
\text { tungen \& Orte }\end{array}$ & $\begin{array}{l}0,060+ \\
(1,81)\end{array}$ & $\begin{array}{l}0,024 \\
(1,41)\end{array}$ & $\begin{array}{l}0,128 * * * \\
(4,25)\end{array}$ & $\begin{array}{l}0,185 * * * \\
(5,66)\end{array}$ \\
\hline Vermeidung lokaler Plätze & $\begin{array}{l}0,172 * * * \\
(5,33)\end{array}$ & $\begin{array}{l}0,072 * * * \\
(4,28)\end{array}$ & $\begin{array}{l}0,184 * * * \\
(6,31)\end{array}$ & $\begin{array}{l}0,191 * * * \\
(5,92)\end{array}$ \\
\hline Kleinstadt & $\begin{array}{l}0,011 \\
(0,14)\end{array}$ & $\begin{array}{l}0,151 \\
(1,12)\end{array}$ & $\begin{array}{l}0,133^{*} \\
(2,36)\end{array}$ & $\begin{array}{l}0,156^{*} \\
(2,32)\end{array}$ \\
\hline Großstadt & $\begin{array}{l}0,024 \\
(0,31)\end{array}$ & $\begin{array}{l}0,070 \\
(1,61)\end{array}$ & $\begin{array}{l}0,134^{*} \\
(1,97)\end{array}$ & $\begin{array}{l}0,137+ \\
(1,90)\end{array}$ \\
\hline Frau & $\begin{array}{l}-0,033 \\
(-1,19)\end{array}$ & $\begin{array}{l}-0,021 \\
(-1,40)\end{array}$ & $\begin{array}{l}-0,010 \\
(-0,40)\end{array}$ & $\begin{array}{l}-0,049+ \\
(-1,87)\end{array}$ \\
\hline Alter & $\begin{array}{l}-0,047 * * * \\
(-5,76)\end{array}$ & $\begin{array}{l}-0,003 \\
(-0,64)\end{array}$ & $\begin{array}{l}-0,004 \\
(-0,55)\end{array}$ & $\begin{array}{l}0,010 \\
(1,21)\end{array}$ \\
\hline Hohe Bildung & $\begin{array}{l}0,064+ \\
(1,90)\end{array}$ & $\begin{array}{l}-0,008 \\
(-0,43)\end{array}$ & $\begin{array}{l}-0,022 \\
(-0,74)\end{array}$ & $\begin{array}{l}-0,025 \\
(-0,77)\end{array}$ \\
\hline Finanzielle Situation & $\begin{array}{l}-0,028 * * \\
(-2,60)\end{array}$ & $\begin{array}{l}-0,013 * \\
(-2,21)\end{array}$ & $\begin{array}{l}-0,027 * * \\
(-2,77)\end{array}$ & $\begin{array}{l}-0,043 \text { *** } \\
(-4,16)\end{array}$ \\
\hline Belästigungserfahrung & & & $\begin{array}{l}0,192^{* * * *} \\
(7,56)\end{array}$ & $\begin{array}{l}0,124 * * * \\
(4,46)\end{array}$ \\
\hline Gewalterfahrung & & & $\begin{array}{l}0,064 \\
(0,91)\end{array}$ & $\begin{array}{l}0,118+ \\
(1,76)\end{array}$ \\
\hline Vertrauen in Regierung & & & $\begin{array}{l}-0,069 * \\
(-2,54)\end{array}$ & $\begin{array}{l}-0,086^{* * *} \\
(-2,74)\end{array}$ \\
\hline Log-Likelihood & $-606,625$ & $-224,252$ & $-446,983$ & $-526,380$ \\
\hline Likelihood Ratio Chi2(16/19) & 247,98 & 66,54 & 337,25 & 322,82 \\
\hline Prob $>$ Chi2 & $p<0,001$ & $p<0,001$ & $p<0,001$ & $p<0,001$ \\
\hline Pseudo- $R^{2}$ & 0,170 & 0,129 & 0,274 & 0,235 \\
\hline$N$ & 1058 & 1055 & 1021 & 1021 \\
\hline
\end{tabular}

Anmerkungen: AME; z-Werte in Klammern; $+p<0,10, * p<0,05, * * p<0,01, * * * p<0,001$ 
Daten nicht ausgeschlossen werden können. Die Besorgnis, dass die Befragten oder ihnen nahestehende Personen in den kommenden 12 Monaten Opfer von Belästigungen oder körperlichen Attacken werden könnten, ist mit $69 \%$ (Belästigungen) und $58 \%$ (körperliche Übergriffe) weitaus stärker ausgeprägt. Etwas mehr als die Hälfte der Befragten haben angegeben, dass sie Dinge tragen oder mit sich führen, die sie in der Öffentlichkeit als Juden erkennbar machen. Auf einer Skala von 1 bis 10 liegt der Mittelwert für die selbsteingeschätzte Religiosität knapp unter dem Skalenmittelpunkt. Mit $31 \%$ schätzt fast ein Drittel der Befragten ihre jüdische Identität als hoch, $46 \%$ als mittel und $23 \%$ als gering ein. Das Erinnern an den Holocaust gehört für $74 \%$ und die Unterstützung Israels für $53 \%$ zur jüdischen Identität.

Etwas mehr als $10 \%$ der Befragten leben in einem Gebiet, in dem mindestens die Hälfte der Menschen ebenfalls jüdisch sind. Auf 33\% der Befragten trifft zu, dass sie mindestens gelegentlich jüdische Orte oder Veranstaltungen meiden, weil sie sich als Juden dort nicht sicher fühlen; $46 \%$ geben an, aus demselben Grund generell bestimmte lokale Orte zu meiden. Mit $23 \%$ vertraut nur eine Minderheit der Befragten darauf, dass die deutsche Regierung Antisemitismus effektiv bekämpft. Die überwiegende Mehrheit (79\%) der Befragten lebt in einer Grossstadt, $51 \%$ geben als Geschlecht ,weiblich“ an und 79\% haben einen höheren Bildungsabschluss. Die subjektive finanzielle Lage ist mit einem Mittelwert von 4,2 an der Skalenmitte orientiert $(1=$, große Schwierigkeiten“ bis $6=$,,sehr leicht über die Runden zu kommen").

\section{Analysen}

Bevor wir nun die im ersten Teil des Beitrags formulierten Vermutungen mit den Daten konfrontieren, wollen wir zunächst noch kurz auf die wahrgenommenen Tätergruppen eingehen (siehe Tab. 2). Diesbezüglich zeigt sich ein recht deutlicher Trend: Von jenen Personen, die Opfer von Belästigung oder körperlicher Gewalt geworden sind (für Vandalismus liegen keine entsprechenden Ergebnisse vor), berichten mit Abstand die meisten, dass die Tat einen islamistischen Hintergrund besessen hätte. Dies gilt für ca. $41 \%$ derer, die per E-Mail oder Textnachrichten, mit Drohanrufen, durch Auflauern, persönliche Kommentare, beleidigende Gesten oder Kommentare im Internet belästigt wurden und für $60 \%$ derer, die Opfer körperlicher Gewalt gewesen sind. Die am zweithäufigsten genannte Tätergruppe bilden Rechtsradikale: $20 \%$ bzw. $17 \%$ berichteten entsprechende Erfahrungen. Aber auch linker Antisemitismus wird von den Befragten erwähnt. 16\% der belästigten und $13 \%$ der körperlich attackierten Personen äußerten entsprechende Erfahrungen. Auffällig ist zudem, dass Beleidigungen und Belästigungen häufig im näheren sozialen Umfeld der Opfer stattzufinden scheinen: In $20 \%$ der Fälle gingen solche Taten laut Wahrnehmung der Betroffenen von Kolleg*innen, Mitschüler*innen bzw. Kommiliton*innen und in $19 \%$ von Bekannten oder Freund*innen aus. Für Gewalttaten trifft dies in weitaus geringerem Maße zu. Diesbezüglich ist allerdings der recht hohe Anteil (21\%) von jugendlichen Täter*innen herauszustellen.

Allerdings wird an dieser Stelle eine der grundlegenden Schwächen der vorliegenden Daten deutlich: Es bleibt unklar, auf welcher Basis und wie genau die 
Befragten die Zuschreibung der Täterschaft vornehmen. Die Befragten hatten die Möglichkeit auf die Frage ,Wer hat Ihnen das angetan?“ eine Mehrfachauswahl aus einer vorgelegten Liste zu treffen, auf der neben den in Tabelle 2 aufgelisteten Gruppen noch so unterschiedliche Kategorien wie „Polizisten“, „Kunden oder Klienten“ oder ,jemand mit einem christlich extremistischen Hintergrund“ auftauchen. Zwar war es auch möglich anzugeben, dass man den Täter nicht beschreiben kann, aber von dieser Möglichkeit machten nur 140 Befragte Gebrauch.

Kommen wir nun zur Überprüfung der in den Hypothesen formulierten Zusammenhänge. In Tabelle 3 berichten wir die Ergebnisse von vier multiplen logistischen Regressionsmodellen zur Diskriminierungserfahrung und Bedrohungswahrnehmung. Die Effekte werden als durchschnittliche marginale Effekte (average marginal effects, AME) berichtet: diese geben den durchschnittlichen Einfluss einer unabhängigen Variable auf die Wahrscheinlichkeit an, von Diskriminierungserfahrungen oder Bedrohungswahrnehmungen zu berichten. Alle Modelle sind gemäß Likelihood-Quotienten-Test hoch signifikant $(p<0,001)$.

Tabelle 3 verdeutlicht zunächst, dass das Tragen von Symbolen, die Befragte als Jüdin/Jude kennzeichnen, die durchschnittliche Wahrscheinlichkeit Opfer von Belästigung zu werden um 21 Prozentpunkte und Opfer von Vandalismus oder körperlichen Übergriffen zu werden um 5 Prozentpunkte erhöht (Hypothese 1). Die Effekte sind statistisch signifikant auf dem 1-Prozentniveau. Während demnach das Tragen von Symbolen die Wahrscheinlichkeit Opfer zu werden erhöht, implizieren die Ergebnisse aber auch, dass Symbole nicht allein ausschlaggebend sind (es ist kein sehr starker Zusammenhang). So können Jüdinnen/Juden auch auf einem anderen Weg, zum Beispiel aus Gesprächen heraus, der Opfergruppe zugeordnet werden. Bei der Bedrohungswahrnehmung ist ebenso ein positiver Effekt zu beobachten, der allerdings schwächer und nicht statistisch signifikant ausfällt. Sich bedroht fühlende Personen verzichten also nicht zwangsläufig auf das Tragen von Symbolen, die sie als Juden erkennbar machen.

Abgesehen vom Modell zu Vandalismus und körperlichen Übergriffen hat die Religiosität der Befragten durchweg einen positiven und signifikanten Effekt auf die Wahrscheinlichkeit der Diskriminierungserfahrung und Bedrohungswahrnehmung (Hypothesen 2 und 2'). Für den angenommenen Einfluss der jüdischen Identität (Hypothesen 3 und 3') finden wir hingegen kaum Belege. Im Modell zur Bedrohungswahrnehmung antisemitischer Gewaltverbrechen deutet sich allerdings ein schwacher Identitäts-Effekt an: die mittlere Wahrscheinlichkeit steigt um 7 Prozentpunkte, wenn Personen sich stark jüdisch fühlen im Vergleich zu Personen, für die dies nur in geringem Maße gilt.

Konträr zur Annahme in Hypothese 4 werden zugewanderte jüdische Personen mit einer geringeren Wahrscheinlichkeit Opfer von Belästigung (9 Prozentpunkte; signifikant auf 1-Prozentniveau), nehmen jedoch, wie in Hypothese 4' wiederum vermutet, eine stärkere Bedrohung durch körperliche Übergriffe wahr (5 Prozentpunkte; signifikant auf 10-Prozentniveau). Der erste Befund könnte sich so erklären, dass diese Personen sich seit weniger als fünf Jahren in Deutschland aufhalten und deshalb einer kürzeren Zeit der Risikosituation ausgesetzt waren als Personen, die nicht zugewandert sind. Gleichzeitig sind Migrant*innen in der Regel eine vulnerable Gruppe. 
Die Daten unterstützen zudem nicht Hypothese 5, wonach jüdische Personen, denen es wichtig ist, die Erinnerung an den Holocaust zu bewahren, höhere Wahrscheinlichkeiten aufweisen, Opfer von Belästigung und Vandalismus bzw. körperlichen Übergriffen zu werden (Hypothesen 5). Allerdings berichten diese Personen deutlich häufiger, sich bedroht zu fühlen (Hypothese 5'). Die mittleren Veränderungsraten liegen in diesem Fall um die 10 Prozentpunkte. Deutliche Indizien finden wir für die Vermutung, dass jüdische Personen, denen es wichtig ist, die Existenz Israels zu verteidigen (Hypothesen 6 und 6'), öfter Opfer einer antisemitischen Straftat werden und sich auch eher bedroht fühlen. Das gilt für Erfahrungen mit Belästigung (11 Prozentpunkte) sowie die wahrgenommene Bedrohung durch Belästigung (7 Prozentpunkte) und Vandalismus bzw. körperliche Übergriffe (10 Prozentpunkte) gleichermaßen. Es trifft jedoch nicht auf die Erfahrung mit körperlichen Übergriffen oder Vandalismus zu.

Was die kontextuellen Faktoren anbetrifft, lassen sich die folgenden Befunde konstatieren: Wenn jüdische Personen in Gegenden wohnen, in denen überwiegend weitere jüdische Personen leben, dann verringert dies die Wahrscheinlichkeit, Opfer von Belästigung (9 Prozentpunkte) oder körperlichen Übergriffen und Vandalismus (5 Prozentpunkte) zu werden (Hypothese 7). Für die Bedrohungswahrnehmung kann ein entsprechender Zusammenhang mit unseren Daten nicht nachgewiesen werden. Ebenfalls nicht gestützt wird unsere Vermutung, dass das Vermeiden von Plätzen und Veranstaltungen, die als bedrohlich wahrgenommen werden, die Wahrscheinlichkeit verringert, Opfer von Beleidigung oder Gewalt zu werden (Hypothesen 8 und 9). Die Effekte sind positiv und im Fall allgemeiner lokaler Orte auch signifikant. Erklärt werden kann dies wohl dadurch, dass hier ein Kausalzusammenhang vorliegt, der von den Querschnittsdaten nicht erfasst wird: Opfer von antisemitischen Straftaten meiden nach der Tat bedrohliche Orte und Veranstaltungen. Gestützt wird diese Vermutung von den Ergebnissen der beiden Modelle zur Bedrohungswahrnehmung. Hier finden wir deutliche Effekte: Jüdische Personen, die sich bedroht fühlen, meiden eher jüdische Veranstaltungen und Orte sowie lokale Plätze, bei denen antisemitische Übergriffe befürchtet werden. Grundsätzlich können wir aber nicht abschließend klären, ob die Vermeidungsstrategie eher Ursache oder Folge von konkreten Bedrohungen oder subjektiven Bedrohungswahrnehmungen ist - zumal viele Übergriffe (v.a. Belästigungen) im persönlichen Umfeld der Betroffenen stattfinden.

Schließlich unterstützen die Befunde in Tabelle 3 die Vermutung, dass Diskriminierungserfahrungen die Bedrohungswahrnehmung verstärken (Hypothese 10). Dies gilt insbesondere für die Erfahrung mit Belästigungen: Der Unterschied bezüglich der durchschnittlichen Wahrscheinlichkeiten liegt hier bei 12 bzw. 19 Prozentpunkten (signifikant auf dem 1-Prozentniveau). Wie erwartet (Hypothese 11) verringert sich zudem die Wahrscheinlichkeit, sich als Jüdin bzw. Jude bedroht zu fühlen, wenn die Person den Eindruck hat, dass die Regierung Antisemitismus effektiv bekämpft (mittlere Veränderungsraten von 7 und 9 Prozentpunkten; signifikant auf dem 5-Prozentniveau).

Neben den theoriegeleiteten Erwartungen zeigen die Befunde, dass insbesondere jüdische Personen, die in der Stadt leben, eher Bedrohungswahrnehmungen äußern als in ländlichen Gebieten wohnende Befragte. Mit Blick auf die sozioökonomische 
Lage deutet sich an, dass je besser die eigene finanzielle Situation von den Interviewten eingeschätzt wird, desto seltener berichten sie von Diskriminierungserfahrungen und Bedrohungswahrnehmungen. Zudem sind es vor allem jüngere Personen, die angeben, Opfer von Belästigungen zu sein.

\section{Fazit und Diskussion}

Der vorliegende Beitrag hat das Gefahrenpotential des Antisemitismus in Deutschland verdeutlicht und systematisch dokumentiert: Erfahrungen mit antisemitischer Gewalt und Vandalismus in den zurückliegenden fünf Jahren wurden von einem nicht zu vernachlässigenden Anteil von $7 \%$ der befragten Jüdinnen und Juden berichtet. Der Wert für Belästigungen liegt bei $44 \%$. Als häufigste wahrgenommene Tatmotive werden islamistische und rechtsextreme Ideologien genannt. Vor allem Personen, die aufgrund des Tragens von Symbolen als Juden erkennbar sind, waren laut Selbstauskunft betroffen und vermeiden gelegentlich oder öfter Plätze in der lokalen Umgebung, weil sie sich dort unsicher fühlen. Wenn die Befragten hingegen in einer mehrheitlich jüdischen Nachbarschaft lebten, sank die berichtete Wahrscheinlichkeit, Opfer von Belästigungen und Gewalttaten zu werden. Belästigungen wurden zudem insbesondere von religiösen und israelsolidarischen Personen berichtet.

Die Befunde bestätigen somit, dass israel-bezogener Antisemitismus einen wichtigen Faktor des aktuellen Bedrohungspotentials darstellt. Er geht, wie die vorliegenden Daten und andere Studien zeigen, häufig auch von islamistischen Tätern aus (Holz und Kiefer 2010) und findet sich sowohl in der rechtsradikalen Bewegung als auch in einigen linken Strömungen (Brosch et al. 2006; Haury 2002). Aufgrund dieser multiplen ideologischen Anschlussfähigkeit kann der Antizionismus als Hauptquelle des aktuellen Judenhasses gelten (vgl. auch Herf 2006; Hirsh 2013).

Dies spiegelt sich ebenfalls in den Ergebnissen zur Bedrohungswahrnehmung wider. Personen, die die Unterstützung Israels als sehr wichtig für ihre jüdische Identität empfinden, fühlen sich auch in stärkerem Maße bedroht. Ähnliches gilt für die subjektive Bedeutung der Holocausterinnerung. Als Reaktion auf die empfundene Bedrohung verzichten die Befragten zwar laut der vorliegenden Daten nicht auf das Tragen von jüdischen Symbolen, aber sie meiden bestimmte Plätze und Veranstaltungen.

Bei den vorliegenden Ergebnissen müssen einige Einschränkungen diskutiert werden. So erlauben, erstens, die Daten zwar Aussagen über Zusammenhänge zwischen Merkmalen, aber nicht über Kausalrichtungen. Dies liegt vor allem daran, dass es sich hier um nicht-experimentelle Querschnittdaten handelt und damit Ursache und Wirkung nicht differenziert werden können, da alle Variablen gleichzeitig erhoben wurden. Zum Beispiel könnte eine Vermeidung von bestimmten Plätzen zu weniger Diskriminierungserfahrungen führen. Es ist aber auch denkbar, dass Diskriminierungserfahrungen die Vermeidung von bestimmten Plätzen zur Folge haben. Allerdings müsste dann im ersten Fall ein negativer und im zweiten Fall ein positiver Effekt vorliegen. Da unsere Ergebnisse einen positiven Zusammenhang zwischen 
Risikovermeidung und der berichteten Erfahrung, Opfer von Vorurteilskriminalität gewesen zu sein, zeigen, ist es plausibler, wenn auch nicht de facto nachweisbar, davon auszugehen, dass die Viktimisierungserfahrung Ursache und nicht Folge des Vermeidungshandelns ist. Paneldaten aber auch qualitative Studien nach dem Vorbild von Zick et al. (2017) könnten jedoch helfen, restliche Zweifel zu beseitigen.

Zweitens handelt es sich um eine Stichprobe, die auf Selbstselektion basiert, sodass die Generalisierbarkeit der Ergebnisse unserer Studie beschränkt ist (etwa mit Blick auf Personen-Merkmale wie Gender, Bildung und Wohnort). Der empirische Test theoretisch hergeleiteter Hypothesen bedarf zwar nicht unbedingt repräsentativer Daten, jedoch wäre es vor allem im Hinblick auf die deskriptiven Befunde durchaus wünschenswert, zufallsbasierte Stichproben zugrunde legen zu können. Denn nur so kann garantiert werden, dass die Grundgesamtheit der in Deutschland lebenden jüdischen Personen angemessen repräsentiert wird. Dies betrifft nicht nur soziö̈konomische Variablen, sondern auch z. B. die berichtete Diskriminierungserfahrung und Bedrohungswahrnehmung: Denn sich bedroht fühlende Menschen oder Oper von Straftaten haben offenkundig eine größere Motivation, an entsprechenden Befragungen teilzunehmen.

Neben Umfragedaten und qualitativen Interviews können Analysen prozessgenerierter Daten, etwa aus der Polizeistatistik, künftig dabei helfen, die Kontexte diskriminierender Handlungen gegen Jüdinnen und Juden in Deutschland noch besser zu verstehen. Doch auch hier ist zu beachten, dass die erfassten Daten nur einen Teil der Realität widerspiegeln. Die Zahlen der FRA-Umfrage verdeutlichen, dass ein erheblicher Teil der antisemitischen Straftaten wohl entweder nicht zur Anzeige gebracht oder nicht als antisemitische Tat registriert wird. Für 2017 wurden beispielweise 38 Fälle antisemitischer Gewalt von den Behörden notiert, wovon 29 (d.h. $76 \%$ ) dem rechten Milieu zugerechnet wurden (Bundesministerium des Innern 2018). Im FRA-Datensatz berichten 3,7\% der 1218 Befragten von körperlichen Angriffen in den letzten 12 Monaten. Rechnet man diesen Anteil allein auf die ca. 98.000 zum Erhebungszeitpunkt als Mitglieder jüdischer Gemeinden gemeldeten Personen hoch, so müssten die tatsächlichen Fallzahlen um ein Vielfaches höher liegen, selbst wenn man die Fälle abzieht, in denen es mehrere Geschädigte derselben Tat gegeben hat, und bedenkt, dass geschädigte Personen aller Wahrscheinlichkeit nach, eher an der Befragung teilnehmen. Insbesondere die unterschiedlichen Werte für den Tathintergrund überraschen diesbezüglich: unsere Ergebnisse legen nahe, dass die Haupttätergruppe aus dem islamistischen Milieu kommt (ca. 60\% der Gewalttaten). In den offiziellen Statistiken existiert eine entsprechende Kategorie nicht, stattdessen werden hier ,ausländische“ sowie ,religiöse Ideologien“ als Motive genannt (Bundesministerium des Innern 2018) - Bezeichnungen, die eine gewisse Hilflosigkeit und Fachgebietsfremdheit auszudrücken scheinen.

Der Widerspruch dürfte sich denn auch teilweise dadurch auflösen, dass viele islamistisch motivierte Straftaten nicht als ,antisemitisch“ deklariert werden. So zum Beispiel geschehen bei dem Brandanschlag von drei Jugendlichen mit palästinensischer Herkunft auf die Wuppertaler Synagoge im Sommer 2014, der sowohl vom Amtsgericht Wuppertal als auch vom Oberlandesgericht in Düsseldorf nicht als antisemitische Tat, sondern als Kritik an Israel eingestuft worden war (vgl. Laurin 2017). Auch aufgrund des Protests gegen das Wuppertaler Urteil sah sich die 
Bundesregierung gezwungen, im September 2017 eine einheitliche Antisemitismusdefinition vorzulegen, die Angriffe ,gegen jüdische Gemeindeinstitutionen oder religiöse Einrichtungen“" als antisemitische Tat kennzeichnen (vgl. Bundesministerium des Inneren 2017).

In Sachen Bedrohung durch Rechtsextremismus deuten die offiziellen Zahlen und die vorliegenden Umfragedaten hingegen in eine ähnliche Richtung. Neonazistische Gruppen und in Online-Netzwerken radikalisierte Einzeltäter mit antisemitischen Verschwörungstheorien stellen nach wie vor eine zentrale Gefahr für Jüdinnen und Juden in Deutschland dar. Dies haben die jüdischen Gemeinden bereits vor den Anschlägen auf die Hallenser Synagoge immer wieder betont, ohne dass ihnen daraufhin der angemessene Schutz zur Verfügung gestellt worden wäre. Sozialwissenschaftliche Studien wie die vorliegende können dazu beitragen, diesen Zustand zu kritisieren und die Sensibilität für die Gefahrenlage zu verstärken, indem sie über die Wahrnehmungen der Betroffenen berichten.

Funding Open Access funding provided by Projekt DEAL.

Open Access Dieser Artikel wird unter der Creative Commons Namensnennung 4.0 International Lizenz veröffentlicht, welche die Nutzung, Vervielfältigung, Bearbeitung, Verbreitung und Wiedergabe in jeglichem Medium und Format erlaubt, sofern Sie den/die ursprünglichen Autor(en) und die Quelle ordnungsgemäß nennen, einen Link zur Creative Commons Lizenz beifügen und angeben, ob Änderungen vorgenommen wurden.

Die in diesem Artikel enthaltenen Bilder und sonstiges Drittmaterial unterliegen ebenfalls der genannten Creative Commons Lizenz, sofern sich aus der Abbildungslegende nichts anderes ergibt. Sofern das betreffende Material nicht unter der genannten Creative Commons Lizenz steht und die betreffende Handlung nicht nach gesetzlichen Vorschriften erlaubt ist, ist für die oben aufgeführten Weiterverwendungen des Materials die Einwilligung des jeweiligen Rechteinhabers einzuholen.

Weitere Details zur Lizenz entnehmen Sie bitte der Lizenzinformation auf http://creativecommons.org/ licenses/by/4.0/deed.de.

\section{Literatur}

Adorno, Theodor W. 1997a. Schuld und Abwehr: Eine qualitative Analyse zum Gruppenexperiment. In Theodor W. Adorno: Gesammelte Schriften, Bd. 9, Hrsg. Rolf Tiedemann, 121-324. Frankfurt a. M.: Suhrkamp.

Adorno, Theodor W. 1997b. Was bedeutet: Aufarbeitung der Vergangenheit? In Theodor W. Adorno: Gesammelte Schriften, Bd. 10.2, Hrsg. Rolf Tiedemann, 555-572. Frankfurt a. M.: Suhrkamp.

Adorno, Theodor W. 1997c. Zur Bekämpfung des Antisemitismus heute. In Theodor W. Adorno: Gesammelte Schriften, Bd. 20.1, Hrsg. Rolf Tiedemann, 360-383. Frankfurt a. M.: Suhrkamp.

Arnold, Sina, und Jana König. 2016. Flucht und Antisemitismus. Erste Hinweise zu Erscheinungsformen von Antisemitismus bei Geflüchteten und mögliche Umgangsstrategien. Qualitative Befragung von Expert_innen und Geflüchteten. Expertise für den Expertenkreis Antisemitismus. Berlin: Berliner Institut für empirische Integrations- und Migrationsforschung (BIM).

Bergmann, Werner, und Rainer Erb. 1986. Kommunikationslatenz, Moral und öffentliche Meinung. Kölner Zeitschrift für Soziologie und Sozialpsychologie 38:223-246.

Beyer, Heiko. 2015. Theorien des Antisemitismus. Eine Systematisierung. Kölner Zeitschrift für Soziologie und Sozialpsychologie 67:573-589.

Brosch, Matthias, Michael Elm, Norman Geißler, Brigitta E. Simbürger, und Oliver von Wrochem (Hrsg.). 2006. Exklusive Solidarität: Linker Antisemitsmus in Deutschland. Vom Idealismus zur Antiglobalisierungsbewegung, 1. Aufl., Berlin: Metropol. 
Bundesministerium des Inneren, für Bau und Heimat. 2017. Kampf gegen Antisemitismus gehört zu unserer Staatsräson. Bundesinnenminister de Maizière und Außenminister Gabriel stellen neue Arbeitsdefinition von Antisemitismus im Kabinett vor. Meldung vom 20.09.2017. http://www.bmi.bund. de/SharedDocs/Kurzmeldungen/DE/2017/09/definition-antisemitismus.html. Letzter Zugriff am 27. April 2020

Bundesministerium des Innern, für Bau und Heimat. 2018. Straf- und Gewaltdaten im Bereich Hasskriminalität 2016 und 2017

Bundesministerium des Innern, für Bau und Heimat. 2019. Politisch Motivierte Kriminalität im Jahr 2018 Bundesweite Fallzahlen

Bundesregierung. 2017. Antwort auf die Kleine Anfrage der Abgeordneten Volker Beck (Köln), Monika Lazar, Irene Mihalic, weiterer Abgeordneter und der Fraktion BÜNDNIS 90/DIE GRÜNEN. BTDrucksache 18/11152.

Coester, Mark. 2015. „Hasskriminalität“. In Viktimisierungsbefragungen in Deutschland. Ziele, Nutzen, Forschungsstand, herausgegeben von Nathalie Guzy, Christoph Birkel, und Robert Mischkowitz, 333-62. Wiesbaden: BKA.

Decker, Oliver, Johannes Kiess, und Elmar Brähler. 2018. Antisemitische Ressentiments in Deutschland: Verbreitung und Ursachen. In Flucht ins Autoritäre. Rechtsextreme Dynamiken in der Mitte der Gesellschaft, Hrsg. Oliver Decker, Elmar Brähler. Gießen: Psychosozial Verlag.

FRA - European Union Agency for Fundamental Rights. 2013. Diskriminierung und Hasskriminalität gegen Juden in den EU-Mitgliedstaaten: Erfahrungen und Wahrnehmungen im Zusammenhang mit Antisemitismus

FRA - European Union Agency for Fundamental Rights. 2018. Experiences and perceptions of anti-semitism. Second survey on discrimination and hate crime against jews in the $E U$

FRA - European Union Agency for Fundamental Rights. 2019. Second survey on discrimination and hate crime against jews in EU member states: technical report

Gerstenfeld, Phyllis B. 2013. Hate crimes. Los Angeles: SAGE.

Gerstenfeld, Phyllis B. 2017. Hate crime. In The Wiley handbook of violence and agression, Hrsg. Peter Sturmey. Hoboken: Wiley.

Hardy, Stevie-Jade, und Neil Chakraborti. 2020. Visible yet invisible: challenges facing hate crime victims. In Blood, threats and fears: the hidden worlds of hate crime victims Palgrave Hate Studies., Hrsg. S.J. Hardy, N. Chakraborti, 11-23. Cham: Springer.

Haury, Thomas. 2002. Antisemitismus von links. Kommunistische Ideologie, Nationalismus und Antizionismus in der frühen DDR. Hamburg: Hamburger Edition.

Heitmeyer, Wilhelm (Hrsg.). 2002. Deutsche Zustände. Folge 1. Frankfurt a. M.: Suhrkamp.

Herf, Jeffrey. 2006. Anti-semitism and anti-zionism in historical perspective: convergence and divergence. London, New York: Routledge Chapman \& Hall.

Hirsh, David. 2013. Hostility to Israel and Antisemitism: toward a sociological approach. Journal for the Study of Antisemitism 5:1401-1422.

Holz, Klaus, und Michael Kiefer. 2010. Islamistischer Antisemitismus Phänomen und Forschungsstand. In Konstellationen des Antisemitismus: Antisemitismusforschung und sozialpädagogische Praxis, Hrsg. W. Stender, G. Follert, und M. Özdogan, 109-137. Wiesbaden: VS.

Laurin, Stefan. 2017. Wuppertal und die Brandstifter. Jüdische Allgemeine vom 9. Jan. 2017.

Marger, Martin N. 2011. Race and ethnic relations: American and global perspectives. Wadsworth: Cengage.

Pettigrew, Thomas F., und Linda R. Tropp. 2011. When groups meet: the dynamics of intergroup contact. London: Psychology Press.

Poliakov, Léon. 1992. Vom Antizionismus zum Antisemitismus. Freiburg: Ca Ira.

Porat, Dina. 2018. „Antisemitism Worldwide 2018 / General Analysis“. 148.

Rabinovici, Doron, Natan Sznaider, und Christian Heilbronn (Hrsg.). 2019. Neuer Antisemitismus?: Fortsetzung einer globalen Debatte. Berlin: Suhrkamp.

Rensmann, Lars. 1998. Kritische Theorie über den Antisemitismus. Studien zu Struktur, Erklärungspotential und Aktualität. Berlin: Argument.

Rensmann, Lars. 2004. Demokratie und Judenbild. Antisemitismus in der politischen Kultur der Bundesrepublik Deutschland. Wiesbaden: VS.

Salzborn, Samuel (Hrsg.). 2019. Antisemitismus seit 9/11: Ereignisse, Debatten, Kontroversen. BadenBaden: Nomos.

Schönbach, Peter. 1961. Reaktionen auf die antisemitische Welle im Winter 1959/60. Frankfurt a. M.: Europäische Verlagsanstalt. 
Sharansky, Natan. 2004. 3D test of anti-semitism: demonization, double standards, delegitimization. Jewish Political Studies Review 16:3-4.

Stafford, Mark C., und Omer R. Galle. 1984. Victimization rates, exposure to risk, and fear of crime. Criminology 22(2):173-185.

Tagesschau. 2019. Zentralrat der Juden zu Halle „Fehlender Polizeischutz ist skandalös“. Tagesschau vom 09.10.2019. https://www.tagesschau.de/inland/reaktionen-schuesse-halle-103.html. Letzter Zugriff am 27. April 2020

Van Kesteren, John. 2016. „Assessing the Risk and Prevalence of Hate Crime Victimization in Western Europe“. International Review of Victimology 22(2):139-160. https://doi.org/10.1177/0269758015627 046.

Zick, Andreas, et al. 2017. Jüdische Perspektiven auf Antisemitismus in Deutschland. Ein Studienbericht für den Expertenrat Antisemitismus. Bielefeld: Institut für interdisziplinäre Konflikt- und Gewaltforschung.

Ziegler, Raphael, und David B. Mitchell. 2003. Aging and fear of crime: an experimental approach to an apparent paradox. Experimental Aging Research 29(2):173-187. 